Advances in Fuzzy Mathematics.

ISSN 0973-533X Volume 12, Number 3 (2017), pp. 577-608

(C) Research India Publications

https://dx.doi.org/10.37622/AFM/12.3.2017.577-608

\title{
Optimization of Welded Beam with Imprecise Load and Stress by Parameterized Intuitionistic Fuzzy Optimization Technique
}

\author{
Mridula Sarkar* \\ Department of Mathematics, Indian Institute of Engineering Science and Technology, \\ Shibpur, P.O.-Botanic Garden, Howrah-711103, West Bengal, India. \\ (*corresponding author) \\ Tapan Kumar Roy \\ Department of Mathematics, Indian Institute of Engineering Science and Technology, \\ Shibpur, P.O.-Botanic Garden, Howrah-711103, West Bengal, India.
}

\begin{abstract}
This paper develops a solution procedure of fuzzy and intuitionistic fuzzy optimization to solve welded beam with inexact co-efficient and resources. Interval approximation method is used here to convert the imprecise coefficient which is a triangular intuitionistic fuzzy number to an interval number. We transform this interval number to a parametric interval valued functional form and then solve this parametric problem by fuzzy and intuitionistic fuzzy optimization technique. Usually interval valued optimization consist of two level mathematical programs but a parametric interval valued optimization in fuzzy and intuitionistic fuzzy environment is direct approach to find the objective function, this is the main advantage. In this paper we have considered a welded beam design with cost of welding as objectives and the maximum shear stress in the weld group, maximum bending stress in the beam, buckling load of the beam and deflection at the tip of a welded steel beam as constraints Numerical example is given here to illustrate this structural model through this approximation method.
\end{abstract}

Keywords: Intuitionistic Fuzzy Set; Generalized Fuzzy Number; Generalized Intuitionistic Fuzzy Number; Nearest Interval Approximation; Interval Valued Function; Single Objective Intuitionistic Optimization; Welded Beam Optimization. 


\section{INTRODUCTION}

Welding, a process of joining metallic parts with the application of heat or pressure or the both, with or without added material, is an economical and efficient method for obtaining permanent joints in the metallic parts. This welded joints are generally used as a substitute for riveted joint or can be used as an alternative method for casting or forging. The welding processes can broadly be classified into following two groups, the welding process that uses heat alone to join two metallic parts and the welding process that uses a combination of heat and pressure for joining (Bhandari. V. B). However, above all the design of welded beam should preferably be economical and durable one. Since decades, deterministic optimization has been widely used in practice for optimizing welded connection design. These include mathematical traditional optimization algorithms (Ragsdell \& Phillips [1]),GA-based methods (Deb [2], Deb [3], Coello [4], Coello [5]), particle swarm optimization (Reddy [6]), harmony search method (Lee \& Geem [7]), and Big-Bang Big-Crunch (BB-BC) algorithm (O. Hasançebi, [8]), subset simulation ( $\mathrm{Li}$ [9]), improved harmony search algorithm (Mahadavi [10]), as methods to solve this problem. All these deterministic optimizations aim to search the optimum solution under given constraints without consideration of uncertainties. So, while a deterministic optimization approach is unable to handle structural performances such as imprecise stresses and deflection etc. due to the presence of impreciseness, to get rid of such problem fuzzy (Zadeh, [11]), intuitionistic fuzzy (Atanassov,[12]) play great roles.

In IFS theory we usually consider degree of acceptance, and degree of rejection where as we consider only membership function in fuzzy set. A few research work has been done on intuitionistic fuzzy optimization in the field of structural optimization. Sarkar [13] optimize two bar truss design with imprecise load and stress in intuitionistic fuzzy environment calculating total integral values of triangular intuitionistic fuzzy number. Shu [14] applied triangular intuitionistic fuzzy number to fault tree analysis on printed board circuit assembly. P.Grzegorzewski et.al [15],H.B.Mitchell et.al [16],G.Nayagam et.al [17],H.M.Nehi et.al [18],S.Rezvani et.al [19] have been employed concept of intuitionistic fuzzy number in multi-attribute decision making(MADM) problem .So indeterminate information should be considered in decision making process. So to deal with different impreciseness on load, stresses and deflection, we have been motivated to incorporate the concept of intuitionistic fuzzy number in this problem, and have developed fuzzy as well as intuitionistic fuzzy optimization algorithm to optimize the optimum design in imprecise environment. We define generalized triangular fuzzy, intuitionistic number and nearest interval approximation of this number and using parametric interval valued function for approximated interval number of triangular fuzzy and intuitionistic fuzzy number we solve a single objective welded beam design problem 
in both fuzzy and intuitionistic fuzzy environment .This paper develops optimization algorithm using max-min operator in fuzzy and intuitionistic fuzzy environment to optimize the cost of welding, while the maximum shear stress in the weld group, maximum bending stress in the beam, and buckling load of the beam have been considered and deflection at the tip of a welded steel beam as constraints. Here parametric interval valued function of generalized triangular fuzzy and intuitionistic fuzzy number have been considered for applied load, stress and deflection .

The present study investigates computational algorithm for solving single-objective nonlinear programming problem by parametric fuzzy and Intuitionistic fuzzy optimization approach. The remainder of this paper is organized in the following manner. In section 2, we discuss about single objective welded beam design. In section 3, we discuss about fuzzy set, fuzzy number, intuitionistic fuzzy set, intuitionistic fuzzy number, $\alpha$-cut and arithmetic operation on triangular intuitionistic fuzzy number. In section 4, we discuss the solution procedure of single objective nonlinear programming problem by parametric fuzzy and intuitionistic non-linear programming technique. In section 5, we discuss about solution of single objective welded beam optimization Problem by parametric fuzzy and intuitionistic fuzzy optimization technique. In section 6, we discuss about numerical solution of single objective welded beam. Finally, we draw conclusions from the results in section 7.

\section{SINGLE-OBJECTIVE STRUCTURAL MODEL}

In sizing optimization problems, the aim is to minimize single objective function, usually the cost of the structure under certain behavioural constraints which are displacement or stresses. The design variables are most frequently chosen to be dimensions of the height, length, depth and width of the structures. Due to fabrications limitations the design variables are not continuous but discrete for belongingness of cross-sections to a certain set. A discrete structural optimization problem can be formulated in the following form

Minimize $C(X)$

subject to $\sigma_{i}(X) \leq\left[\sigma_{i}\right], i=1,2, \ldots ., m$

$X_{j} \in R^{d}, \quad j=1,2, \ldots . ., n$

where $C(X)$ represents cost function, $\sigma_{i}(X)$ is the behavioural constraints and $\left[\sigma_{i}(X)\right]$ denotes the maximum allowable value , ' $m$ ' and ' $n$ ' are the number of constraints and design variables respectively. A given set of discrete value is expressed by $R^{d}$ and in this paper objective function is taken as 


$$
C(X)=\sum_{t=1}^{T} c_{t} \prod_{n=1}^{m} x_{n}^{t n}
$$

and constraint are chosen to be stress of structures as follows

$\sigma_{i}(X) \lesssim \sigma_{i}$ with allowable tolerance $\sigma_{i}^{0}$ for $i=1,2, \ldots, m$

And deflection of the structure as follows

$\delta(X) \lesssim \delta_{\max }$ with allowable tolerance $\delta_{\max }^{0}$

Where $c_{t}$ is the cost coefficient of $\mathrm{t}^{\text {th }}$ side and $x_{n}$ is the $n^{\text {th }}$ design variable respectively, $m$ is the number of structural element, $\sigma_{i}$ and $\sigma_{i}^{0} \delta_{\max }^{0}$ are the $i^{\text {th }}$ stress , allowable stress and allowable deflection respectively.

\section{MATHEMATICAL PRELIMINARIES}

\subsection{Fuzzy Set}

Let $X$ denotes a universal set. Then the fuzzy subset $A$ in $X$ is a subset of order pairs $\tilde{A}=\left\{\left(x, \mu_{\tilde{A}}(x)\right): x \in X\right\}$ where $\mu_{\tilde{A}}: X \rightarrow[0,1]$ is called the membership function which assigns a real number $\mu_{\tilde{A}}(x)$ in the interval $[0,1]$ to each element $x \in X . A$ is non-fuzzy and $\mu_{\tilde{A}}(x)$ is identical to the characteristic function of crisp set.It is clear that the range of membership function is a subset of non-negative real numbers.

\section{2. $\alpha$ - Level set or $\alpha-$ cut of a Fuzzy Set}

The $\alpha$-level set of a fuzzy set $A$ of $X$ is a crisp set $A_{\alpha}$ which contains all the elements of $X$ that have membership values greater than or equal to $\alpha$ i.e $A=\left\{x: \mu_{A}(x) \geq \alpha, x \in X, \alpha \in[0,1]\right\}$.

\subsection{Generalized Fuzzy Number}

A generalised fuzzy number $\tilde{A}=(a, b, c, d ; w)$ where $0<w \leq 1$ and $a, b, c, d$ are real numbers. The generalized fuzzy number $\tilde{A}=(a, b, c, d ; w)$ is a fuzzy subset of real line $\square$ whose membership function $\mu_{\tilde{A}}(x)$ satisfies the following conditions

i) $\quad \mu_{\tilde{A}}(x)$ is a continuous mapping from $\square$ to closed interval $[0,1]$. 
ii) $\mu_{\tilde{A}}(x)=0$ where $-\infty<x \leq a$

ii) $\mu_{\tilde{A}}(x)$ is strictly increasing in constant rate on $[a, b]$

iv) $\mu_{\tilde{A}}(x)=w$ where $b \leq x \leq c$.

v) $\mu_{\tilde{A}}(x)$ is strictly decreasing in constant rate on $[c, d]$

vi) $\mu_{\tilde{A}}=0$ where $d \leq x \leq \infty$

Now if $b=c, w=1$ it is called triangular fuzzy number $(\mathrm{TFN})$ as $\tilde{A}=(a, b, d ; w)$ and membership function of fuzzy set $\tilde{A}=\left(x, \mu_{\tilde{A}}(x)\right)$ can be defined as $\mu_{\tilde{A}}(x)=\left\{\begin{array}{cl}w\left(\frac{x-a}{b-a}\right) & \text { for } a \leq x \leq b \\ w\left(\frac{d-x}{d-b}\right) & \text { for } b \leq x \leq d \\ 0 & \text { otherwise }\end{array}\right.$

\section{4. $\quad \alpha-$ Level set or $\alpha-$ cut of a Fuzzy Number}

Let $\tilde{A}=\left(a_{1}^{\mu}, a_{2}, a_{3}^{\mu} ; w_{a}\right)$ be a triangular fuzzy number then $\alpha$-cut of this fuzzy number is defined by the crisp set $A_{\alpha}=\left\{x: \mu_{\tilde{A}}(x) \geq \alpha, x \in X, \alpha \in[0,1]\right\} . A_{\alpha}$ is nonempty bounded closed interval contained in $X$ and it can be denoted by $A_{\alpha}=\left[A_{L}(\alpha), A_{R}(\alpha)\right], A_{L}(\alpha)$ and $A_{R}(\alpha)$ are the lower and upper bound of the closed interval, respectively.

\subsection{Intuitionistic Fuzzy Set}

Let $X=\left\{x_{1}, x_{2}, \ldots ., x_{n}\right\}$ be a finite universal set. An intuitionistic fuzzy set (IFS) set $\tilde{A}^{i}$ in the sense of Atanassov (1986) is given by equation $\tilde{A}^{i}=\left\{<X, \mu_{\tilde{A}^{i}}(x), v_{\tilde{A}^{i}}(x)>\mid x_{i} \in X\right\} \quad$ where the function $\quad \mu_{\tilde{A}^{i}}\left(x^{i}\right): X \rightarrow[0,1]$; $x_{i} \in X \rightarrow \mu_{\tilde{A}^{i}}\left(x_{i}\right) \in[0,1]$ and $\quad v_{\tilde{A}^{i}}\left(x^{i}\right): X \rightarrow[0,1] ; x_{i} \in X \rightarrow v_{\tilde{A}^{i}}\left(x_{i}\right) \in[0,1]$ define the degree of membership and degree of non-membership of an element $x_{i} \in X$ to the set $\tilde{A}^{i} \subseteq X$, such that they satisfy the condition $0 \leq \mu_{\tilde{A}^{i}}\left(x_{i}\right)+v_{\tilde{A}^{i}}\left(x_{i}\right) \leq 1, \forall x_{i} \in X$. For 
each IFS $\tilde{A}^{i}$ in $X$ the amount $\Pi_{\tilde{A}^{i}}\left(x_{i}\right)=1-\left(\mu_{\tilde{A}^{i}}\left(x^{i}\right)+v_{\tilde{A}^{i}}\left(x^{i}\right)\right)$ is called the degree of uncertainty (or hesitation ) associated with the membership of elements $x_{i} \in X$ in $\tilde{A}^{i}$ we call it intuitionistic fuzzy index of $\tilde{A}^{i}$ with respect of an element $x_{i} \in X$.

\subsection{Generalized Intuitionistic Fuzzy Number}

A generalised intuitionistic fuzzy number $\tilde{A}^{i}$ can be defined as with the following properties

i) It is an intuitionistic fuzzy subset of real line.

ii) It is normal i.e there is any $x_{0} \in R$ such that $\mu_{\tilde{A}^{i}}\left(x_{0}\right)=w(\in R)$ and $v_{\tilde{A}^{i}}\left(x_{0}\right)=\tau(\in R)$ for $w+\tau \leq 1$;

iii) It is a convex set for membership function $\mu_{\tilde{A}^{i}}(x)$ i.e

$$
\mu_{\tilde{A}^{i}}\left(\lambda x_{1}+(1-\lambda) x_{2}\right) \geq \min \left(\mu_{\tilde{A}^{i}}\left(x_{1}\right), \mu_{\tilde{A}^{i}}\left(x_{2}\right)\right) \text { for all } x_{1}, x_{2} \in R, \lambda \in[0, w] \text {. }
$$

iv) It is a concave set for membership function $\mu_{\tilde{A}^{i}}(x)$ i.e

$$
\mu_{\tilde{A}^{i}}\left(\lambda x_{1}+(1-\lambda) x_{2}\right) \geq \max \left(\mu_{\tilde{A}^{i}}\left(x_{1}\right), \mu_{\tilde{A}^{i}}\left(x_{2}\right)\right) \text { for all } x_{1}, x_{2} \in R, \lambda \in[\tau, 1] \text {. }
$$

v) $\mu_{\tilde{\tilde{A}}^{i}}$ is continuous mapping from $R$ to the closed interval $[0, w]$ and $v_{\tilde{A}^{i}}$ is continuous mapping from $R$ to the closed interval $[\tau, 1]$ and for $x_{0} \in R$ the relation $\mu_{\tilde{A}^{i}}+v_{\tilde{A}^{i}} \leq 1$ holds.

\subsection{Generalized Triangular Intuitionistic Fuzzy Number}

A generalized triangular intuitionistic fuzzy number $\tilde{A}^{i}=\left(\left(a_{1}^{\mu}, a_{2}, a_{3}^{\mu} ; w_{a}\right)\left(a_{1}^{v}, a_{2}, a_{3}^{\nu} ; \tau_{a}\right)\right)$ is a IFN in $\mathrm{R}$ and can be defined with the following membership function and non-membership function as follows 


$$
\begin{aligned}
& \mu_{\tilde{A}^{i}}=\left\{\begin{array}{cl}
w_{a} \frac{x-a_{1}^{\mu}}{a_{2}-a_{1}^{\mu}} & \text { for } a_{1}^{\mu} \leq x \leq a_{2} \\
w_{a} & \text { for } x=a_{2} \\
w_{a} \frac{a_{3}^{\mu}-x}{a_{3}^{\mu}-a_{2}} & \text { for } a_{2} \leq x \leq a_{3}^{\mu} \\
0 & \text { otherwise }
\end{array}\right. \\
& v_{\tilde{A}^{i}}=\left\{\begin{array}{cl}
\tau_{a} \frac{x-a_{1}^{v}}{a_{2}-a_{1}^{v}} & \text { for } a_{1}^{v} \leq x \leq a_{2} \\
\tau_{a} \frac{x-a_{2}}{a_{3}^{v}-a_{2}} & \text { for } x=a_{2} \leq x \leq a_{3}^{v} \\
1 & \text { otherwise }
\end{array}\right.
\end{aligned}
$$

Where $a_{1}^{v} \leq a_{1}^{\mu} \leq a_{2} \leq a_{3}^{\mu} \leq a_{3}^{v}$.

\section{8. $\quad \alpha$ - Level set or $\alpha-$ cut of a Intuitionistic Fuzzy Number}

Let $\tilde{A}^{i}=\left(\left(a_{1}^{\mu}, a_{2}, a_{3}^{\mu} ; w_{a}\right)\left(a_{1}^{v}, a_{2}, a_{3}^{v} ; \tau_{a}\right)\right)$ be a triangular intuitionistic fuzzy number then $\alpha$-cut of this fuzzy number is defined by the closed interval $\left[\mu_{A_{L}^{i}}(\alpha), \mu_{A_{U}^{i}}(\alpha)\right], \alpha \in(0,1]$ and $\quad\left[v_{A_{L}^{i}}(\alpha), v_{A_{U}^{i}}(\alpha)\right], \alpha \in[0,1)$ where $\mu_{A_{L}^{i}}(\alpha)=\inf \left\{x \in R: \mu_{A_{L}^{i}}(x) \geq \alpha\right\}$ $\mu_{A_{U}^{i}}(\alpha)=\sup \left\{x \in R: \mu_{A_{U}^{i}}(x) \geq \alpha\right\}, v_{A_{L}^{i}}(\alpha)=\inf \left\{x \in R: v_{A_{L}^{i}}(x) \leq \alpha\right\}$ $v_{A_{U}^{i}}(\alpha)=\sup \left\{x \in R: v_{A_{U}^{i}}(x) \leq \alpha\right\}$,

\subsection{Arithmatic Operations on Triangular Intuitionistic Fuzzy Numbers}

Let $\tilde{A}^{i}=\left(\left(a_{1}^{\mu}, a_{2}, a_{3}^{\mu} ; w_{a}\right)\left(a_{1}^{v}, a_{2}, a_{3}^{v} ; \tau_{a}\right)\right) \quad$ and $\quad \tilde{B}^{i}=\left(\left(b_{1}^{\mu}, b_{2}, b_{3}^{\mu} ; w_{b}\right)\left(b_{1}^{v}, b_{2}, b_{3}^{v} ; \tau_{b}\right)\right)$ be two triangular intuitionistic fuzzy number then the arithmetic operations on these numbers can be defined as follows

(i)

$$
\tilde{A}^{i}+\tilde{B}^{i}=\left(\left(a_{1}^{\mu}+b_{1}^{\mu}, a_{2}+b_{2}, a_{3}^{\mu}+b_{3}^{\mu} ; \min \left(w_{a}, w_{b}\right)\right)\left(a_{1}^{v}+b_{1}^{v}, a_{2}+b_{2}, a_{3}^{v}+b_{3}^{v} ; \max \left(\tau_{a}, \tau_{b}\right)\right)\right)
$$


(ii) $\tilde{A}^{i}-\tilde{B}^{i}=\left(\left(a_{1}^{\mu}-b_{1}^{\mu}, a_{2}-b_{2}, a_{3}^{\mu}-b_{3}^{\mu} ; \min \left(w_{a}, w_{b}\right)\right)\left(a_{1}^{v}-b_{1}^{v}, a_{2}-b_{2}, a_{3}^{v}-b_{3}^{v} ; \max \left(\tau_{a}, \tau_{b}\right)\right)\right)$

(iii) $k \tilde{A}^{i}= \begin{cases}\left(\left(k a_{1}^{\mu}, k a_{2}, k a_{3}^{\mu} ; w_{a}\right)\left(k a_{1}^{v}, k a_{2}, k a_{3}^{v} ; \tau_{a}\right)\right) & \text { for } k>0 \\ \left(\left(k a_{3}^{\mu}, k a_{2}, k a_{1}^{\mu} ; w_{a}\right)\left(k a_{3}^{v}, k a_{2}, k a_{1}^{v} ; \tau_{a}\right)\right) & \text { for } k<0\end{cases}$

(iv) $\tilde{A}^{i} \cdot \tilde{B}^{i}= \begin{cases}\left(\left(a_{1}^{\mu} b_{1}^{\mu}, a_{2} b_{2}, a_{3}^{\mu} b_{3}^{\mu} ; \min \left(w_{a}, w_{b}\right)\right)\left(a_{1}^{v} b_{1}^{v}, a_{2} b_{2}, a_{3}^{v} b_{3}^{v} ; \max \left(\tau_{a}, \tau_{b}\right)\right)\right) & \text { for } \tilde{A}^{i}>0, \tilde{B}^{i}>0 \\ \left(\left(a_{1}^{\mu} b_{3}^{\mu}, a_{2} b_{2}, a_{3}^{\mu} b_{1}^{\mu} ; \min \left(w_{a}, w_{b}\right)\right)\left(a_{1}^{v} b_{3}^{v}, a_{2} b_{2}, a_{3}^{v} b_{1}^{v} ; \max \left(\tau_{a}, \tau_{b}\right)\right)\right) & \text { for } \tilde{A}^{i}>0, \tilde{B}^{i}<0 \\ \left(\left(a_{3}^{\mu} b_{3}^{\mu}, a_{2} b_{2}, a_{1}^{\mu} b_{1}^{\mu} ; \min \left(w_{a}, w_{b}\right)\right)\left(a_{3}^{v} b_{3}^{v}, a_{2} b_{2}, a_{1}^{v} b_{1}^{v} ; \max \left(\tau_{a}, \tau_{b}\right)\right)\right) & \text { for } \tilde{A}^{i}<0, \tilde{B}^{i}<0\end{cases}$

$\left(\left(\left(a_{1}^{\mu} / b_{3}^{\mu}, a_{2} / b_{2}, a_{3}^{\mu} / b_{1}^{\mu} ; \min \left(w_{a}, w_{b}\right)\right)\left(a_{1}^{v} / b_{3}^{v}, a_{2} / b_{2}, a_{3}^{v} / b_{1}^{v} ; \max \left(\sigma_{a}, \sigma_{b}\right)\right)\right)\right.$ for $\tilde{A}^{i}>0, \tilde{B}^{i}>0$

(v) $\tilde{A}^{i} / \tilde{B}^{i}=\left\{\left(\left(a_{3}^{\mu} / b_{3}^{\mu}, a_{2} / b_{2}, a_{1}^{\mu} / b_{1}^{\mu} ; \min \left(w_{a}, w_{b}\right)\right)\left(a_{3}^{v} / b_{3}^{v}, a_{2} / b_{2}, a_{1}^{v} / b_{1}^{v} ; \max \left(\sigma_{a}, \sigma_{b}\right)\right)\right)\right.$ for $\tilde{A}^{i}<0, \tilde{B}^{i}>0$ $\left(\left(a_{3}^{\mu} / b_{1}^{\mu}, a_{2} / b_{2}, a_{1}^{\mu} / b_{3}^{\mu} ; \min \left(w_{a}, w_{b}\right)\right)\left(a_{3}^{v} / b_{1}^{v}, a_{2} / b_{2}, a_{1}^{v} / b_{3}^{v} ; \max \left(\sigma_{a}, \sigma_{b}\right)\right)\right)$ for $\tilde{A}^{i}<0, \tilde{B}^{i}<0$

\section{MATHEMATICAL ANALYSIS}

\subsection{Nearest Interval Approximation for Fuzzy Number}

Here we want to approximate an fuzzy number $\tilde{A}=\left(a_{1}^{\mu}, a_{2}, a_{3}^{\mu} ; w_{a}\right)$ by a crisp model.

Let $\tilde{A}$ and $\tilde{B}$ be two fuzzy number with $\alpha$ - cuts $\operatorname{are}\left[A_{L}(\alpha), A_{U}(\alpha)\right]$ and $\left[B_{L}(\alpha), B_{U}(\alpha)\right]$ respectively.Then the distance between them can be measured according to Euclidean matric (Dey $\tilde{d}_{E}^{2}=\int_{0}^{1}\left(A_{L}(\alpha)-B_{L}(\alpha)\right)^{2} d \alpha+\int_{0}^{1}\left(A_{U}(\alpha)-B_{U}(\alpha)\right)^{2} d \alpha$

Now we find a closed interval $\tilde{C}_{d_{E}}\left(\tilde{A}^{i}\right)=\left[C_{L}, C_{U}\right]$ which is nearest to $\tilde{A}$ with respect to the matric $\tilde{d}_{E}$.Again it is obvious that each real interval can also be considered as an fuzzy number with constant $\alpha$-cut $\left(\tilde{C}_{d_{E}}(\tilde{A})\right) \alpha=\left[C_{L}, C_{U}\right]$ for all $\alpha \in[0,1]$.Now we have to minimize $\tilde{d}_{E}^{2}\left(\tilde{A}, \tilde{C}_{d_{E}}(\tilde{A})\right)=\int_{0}^{1}\left(A_{L}(\alpha)-C_{L}\right)^{2} d \alpha+\int_{0}^{1}\left(A_{U}(\alpha)-C_{U}\right)^{2} d \alpha$ with respect to $C_{L}$ and $C_{R}$.In order to minimize $\tilde{d}_{E}\left(\tilde{A}, \tilde{C}_{d_{E}}(\tilde{A})\right)$ 
It is sufficient to minimize the functions $D\left(C_{L}, C_{U}\right)\left(=\tilde{d}_{E}^{2}\left(\tilde{A}, \tilde{C}_{d_{E}}(\tilde{A})\right)\right)$. The first partial derivatives are $\frac{\partial D\left(C_{L}, C_{R}\right)}{\partial C_{L}}=-2 \int_{0}^{1} A_{L}(\alpha) d \alpha+2 C_{L}$

$\frac{\partial D\left(C_{L}, C_{U}\right)}{\partial C_{U}}=-2 \int_{0}^{1} A_{U}(\alpha) d \alpha+2 C_{U}$

And then we solve the system $\frac{\partial D\left(C_{L}, C_{U}\right)}{\partial C_{U}}=0, \frac{\partial D\left(C_{L}, C_{U}\right)}{\partial C_{U}}=0$ The solution is $C_{L}=\int_{0}^{1} A_{L}(\alpha) d \alpha ; C_{U}=\int_{0}^{1} A_{U}(\alpha) d \alpha$

Since det $\left(\begin{array}{ll}\frac{\partial^{2} d\left(C_{L}, C_{U}\right)}{\partial C_{L}^{2}} & \frac{\partial^{2} D\left(C_{L}, C_{U}\right)}{\partial C_{L} \partial C_{U}} \\ \frac{\partial^{2} D\left(C_{L}, C_{U}\right)}{\partial C_{L} \partial C_{U}} & \frac{\partial^{2} F_{1}\left(C_{L}, C_{U}\right)}{\partial C_{U}^{2}}\end{array}\right)$

$=\left(\begin{array}{ll}2 & 0 \\ 0 & 2\end{array}\right)=4>0$ then $C_{L} C_{U}$ mentioned above minimize $D\left(C_{L}, C_{U}\right)$. The nearest interval of the intuitionistic fuzzy number $\tilde{A}$ with respect to the matric $\tilde{d}_{E}$ is $\tilde{C}_{d_{E}}(\tilde{A})=\left[\int_{0}^{1} A_{L}(\alpha) d \alpha, \int_{0}^{1} A_{U}(\alpha) d \alpha\right]=\left[\frac{(2 w-1) a_{1}+a_{2}}{2 w}, \frac{(2 w-1) a_{3}+a_{2}}{2 w}\right]$

\subsection{Nearest Interval Approximation for Intuitionistic Fuzzy Number}

Here we want to approximate an intuitionistic fuzzy number $\tilde{A}^{i}=\left(\left(a_{1}^{\mu}, a_{2}, a_{3}^{\mu} ; w_{a}\right)\left(a_{1}^{\nu}, a_{2}, a_{3}^{\nu} ; \eta_{a}\right)\right)$ by a crisp model.

Let $\tilde{A}^{i}$ and $\tilde{B}^{i}$ be two intuitionistic fuzzy number.Then the distance between them can be measured according to Euclidean matric as (Dey [22]) $\tilde{d}_{E}^{2}=\frac{1}{2} \int_{0}^{1}\left(\mu_{A_{L}}(\alpha)-\mu_{B_{L}}(\alpha)\right)^{2} d \alpha+\frac{1}{2} \int_{0}^{1}\left(\mu_{A_{U}}(\alpha)-\mu_{B_{U}}(\alpha)\right)^{2} d \alpha$ $+\frac{1}{2} \int_{0}^{1}\left(v_{A_{L}}(\alpha)-v_{B_{L}}(\alpha)\right)^{2} d \alpha+\frac{1}{2} \int_{0}^{1}\left(v_{A_{U}}(\alpha)-v_{B_{U}}(\alpha)\right)^{2} d \alpha$ 
Now we find a closed interval $\tilde{C}_{d_{E}}\left(\tilde{A}^{i}\right)=\left[C_{L}, C_{U}\right]$ which is nearest to $\tilde{A}^{i}$ with respect to the matric $\tilde{d}_{E}$.Again it is obvious that each real interval can also be considered as an intuitionistic fuzzy number with constant $\alpha$-cut $\left[C_{L}, C_{U}\right]$ for all $\alpha \in[0,1]$.Now we have to minimize $\tilde{d}_{E}\left(\tilde{A}^{i}, \tilde{C}_{d_{E}}\left(\tilde{A}^{i}\right)\right)$ with respect to $C_{L}$ and $C_{U}$, that is to minimize

$$
\begin{aligned}
& F_{1}\left(C_{L}, C_{U}\right)=\int_{0}^{1}\left(\mu_{A_{L}}(\alpha)-C_{L}\right)^{2} d \alpha+\int_{0}^{1}\left(\mu_{A_{U}}(\alpha)-C_{U}\right)^{2} d \alpha \\
& +\int_{0}^{1}\left(v_{A_{L}}(\alpha)-C_{L}\right)^{2} d \alpha+\int_{0}^{1}\left(v_{A_{U}}(\alpha)-C_{U}\right)^{2} d \alpha
\end{aligned}
$$

With respect to $C_{L}$ and $C_{U}$. We define partial derivatives

$$
\begin{aligned}
& \frac{\partial F_{1}\left(C_{L}, C_{U}\right)}{\partial C_{L}}=-2 \int_{0}^{1}\left(\mu_{A_{L}}(\alpha)+v_{A_{L}}(\alpha)\right) d \alpha+4 C_{L} \\
& \frac{\partial F_{1}\left(C_{L}, C_{U}\right)}{\partial C_{U}}=-2 \int_{0}^{1}\left(\mu_{A_{U}}(\alpha)+v_{A_{U}}(\alpha)\right) d \alpha+4 C_{U}
\end{aligned}
$$

And then we solve the system

$$
\begin{aligned}
& \frac{\partial F_{1}\left(C_{L}, C_{U}\right)}{\partial C_{L}}=0, \frac{\partial F_{1}\left(C_{L}, C_{U}\right)}{\partial C_{U}}=0 \\
& C_{L}=\int_{0}^{1} \frac{\mu_{A_{L}}(\alpha)+v_{A_{L}}(\alpha)}{2} d \alpha ; C_{U}=\int_{0}^{1} \frac{\mu_{A_{U}}(\alpha)+v_{A_{U}}(\alpha)}{2} d \alpha \\
& \operatorname{det}\left(\begin{array}{ll}
\frac{\partial^{2} F_{1}\left(C_{L}, C_{U}\right)}{\partial C_{L}^{2}} & \frac{\partial^{2} F_{1}\left(C_{L}, C_{U}\right)}{\partial C_{L} \partial C_{U}} \\
\text { Since } & \left.\frac{\partial^{2} F_{1}\left(C_{L}, C_{U}\right)}{\partial C_{U} \partial C_{L}}, C_{U}\right) \\
\partial C_{U}^{2}
\end{array}\right)
\end{aligned}
$$

$=\left(\begin{array}{ll}4 & 0 \\ 0 & 4\end{array}\right)=4>0$ then $C_{L} C_{U}$ mentioned above minimize $F_{1}\left(C_{L}, C_{U}\right)$. The nearest interval of the intuitionistic fuzzy number $\tilde{A}^{i}$ with respect to the matric $\tilde{d}_{E}$ is

$$
\begin{aligned}
& \tilde{C}_{d_{E}}\left(\tilde{A}^{i}\right)=\left[\int_{0}^{1} \frac{\mu_{A_{L}}(\alpha)+v_{A_{L}}(\alpha)}{2} d \alpha, \int_{0}^{1} \frac{\mu_{A_{U}}(\alpha)+v_{A_{U}}(\alpha)}{2} d \alpha\right] \\
& =\left[\frac{a_{1}^{\mu}+a_{2}}{2}+\frac{a_{2}-a_{1}^{\mu}}{4 w}+\frac{a_{2}-a_{1}^{\nu}}{4 \eta}, \frac{a_{2}+a_{3}^{\mu}}{2}+\frac{a_{3}^{\mu}-a_{2}}{4 w}+\frac{a_{3}^{v}-a_{2}}{4 \eta}\right]
\end{aligned}
$$




\subsection{Parametric Interval valued Function}

If $[m, n]$ be an interval with $m, n>0$ we can express an interval number by a function. The parametric interval-valued function for the interval $[m, n]$ can be taken as $g(s)=m^{1-s} n^{s}$ for $s \in[0,1]$ which is strictly monotone continuous function and its inverse exists .Let $\psi$ be the inverse of $g(s)$ then $s=\frac{\log \psi-\log m}{\log n-\log m}$.

\subsection{Formulation of Fuzzy and Intuitionistic Fuzzy Programming with imprecise coefficient in parametric form}

A single objective intuitionistic fuzzy non-linear programming problem with imprecise co-efficient can be formulated as

Minimize $\tilde{f}(x)=\sum_{t=1}^{T} \xi_{t} \tilde{c}_{t} \prod_{j=1}^{n} x_{j}^{a_{i j}}$

Such that $\tilde{f}_{i}(x)=\sum_{t=1}^{T_{i}} \xi_{i t} \tilde{c}_{i t} \prod_{j=1}^{n} x_{j}^{a_{i j}} \leq \xi_{i} \tilde{b}_{i}$ for $i=1,2, \ldots ., m$

$$
x_{j}>0 \quad j=1,2, \ldots, n
$$

Here $\xi_{t}, \xi_{i t}, \xi_{i}$ are the signum function used to indicate sign of term in the equation. $\tilde{c}_{t}>0, \tilde{c}_{i t}>0 . a_{t j}, a_{i t j}$ are real numbers for all $i, t, j$.

Here $\quad \tilde{c}_{t}=\left(c_{t}^{1 \mu}, c_{t}^{2}, c_{t}^{3 \mu} ; w_{t}\right) ; \tilde{c}_{i t}=\left(c_{i t}^{1 \mu}, c_{i t}^{2}, c_{i t}^{3 \mu} ; w_{i t}\right) ; \tilde{b}_{i}=\left(b_{i}^{1 \mu}, b_{i}^{2}, b_{i}^{3 \mu} ; w_{i}\right) \quad$ for $\quad$ fuzzy number as coefficients and $\quad \tilde{c}_{t}=\left(\left(c_{t}^{1 \mu}, c_{t}^{2}, c_{t}^{3 \mu} ; w_{t}\right)\left(c_{t}^{1 v}, c_{t}^{2}, c_{t}^{3 v} ; \tau_{t}\right)\right)$; $\tilde{c}_{i t}=\left(\left(c_{i t}^{1 \mu}, c_{i t}^{2}, c_{i t}^{3 \mu} ; w_{i t}\right)\left(c_{i t}^{1 v}, c_{i t}^{2}, c_{i t}^{3 v} ; \tau_{i t}\right)\right) ; \tilde{b}_{i}=\left(\left(b_{i}^{1 \mu}, b_{i}^{2}, b_{i}^{3 \mu} ; w_{i}\right)\left(b_{i}^{1 v}, b_{i}^{2}, b_{i}^{3 v} ; \tau_{i}\right)\right) \quad$ for intuitionistic fuzzy coefficient.

Using nearest interval approximation method for both fuzzy and intuitionistic fuzzy number, we transform all the triangular intuitionistic fuzzy number into interval number i.e $\left[c_{t}^{L}, c_{t}^{U}\right],\left[c_{i t}^{L}, c_{i t}^{U}\right]$, and $\left[b_{i}^{L}, b_{i}^{U}\right]$

Now the intuitionistic single objective programming with imprecise parameter is of the following form

Minimize $\hat{f}(x)=\sum_{t=1}^{T} \xi_{k_{0}} \hat{c}_{t} \prod_{j=1}^{n} x_{j}^{a_{t_{j}}}$ 
Such that $\hat{f}_{i}(x)=\sum_{i=1}^{T_{i}} \xi_{i t} \hat{c}_{i t} \prod_{j=1}^{n} x_{j}^{a_{i j}} \leq \sigma_{i} \hat{b}_{i}$ for $i=1,2, \ldots, m$

$$
x_{j}>0 \quad j=1,2, \ldots ., n
$$

Here $\xi_{t}, \xi_{i t}, \xi_{i}$ are the signum function used to indicate sign of term in the equation. $\hat{c}_{t}>0, \hat{c}_{i t}>0 ; \hat{b}_{i}>0$ denote the interval component i.e $\hat{c}_{t}=\left[c_{t}^{L}, c_{t}^{U}\right], \hat{c}_{i t}=\left[c_{i t}^{L}, c_{i t}^{U}\right]$, and $\hat{b}_{i}=\left[b_{i}^{L}, b_{i}^{U}\right]$ and $a_{t j}, a_{i t j}$ are real numbers for all $i, t, j$.

Using parametric interval valued function the above problem transform into

Minimize $f(x ; s)=\sum_{t=1}^{T} \xi_{t}\left(c_{t}^{L}\right)^{1-s}\left(c_{t}^{U}\right)^{s} \prod_{j=1}^{n} x_{j}^{a_{t j}}$

Such that $f_{i}(x ; s)=\sum_{t=1}^{T_{i}} \xi_{i t}\left(c_{i t}^{L}\right)^{1-s}\left(c_{i t}^{U}\right)^{s} \prod_{j=1}^{n} x_{j}^{a_{i j}} \leq \xi_{i}\left(b_{i}^{L}\right)^{1-s}\left(b_{i}^{U}\right)^{s}$ for $i=1,2, \ldots ., m$

$$
x_{j}>0 \quad j=1,2, \ldots ., n s \in[0,1]
$$

Here $\xi_{t}, \xi_{i t}, \xi_{i}$ are the signum function used to indicate sign of term in the equation.

This is a parametric single objective non-linear programming problem and can be solved by intuitionistic fuzzy optimization technique.

\subsection{Fuzzy and Intuitionistic Fuzzy Non-linear Programming (IFNLP) Optimization to solve Parametric Single-Objective Non-linear Programming Problem (PSONLP)}

Let us consider a single-objective parametric nonlinear optimization problem as

Minimize $f(x)$

$g_{j}(x ; s) \leq b_{j}(s) \quad j=1,2, \ldots \ldots, m$

$x \geq 0 ; s \in[0,1]$

Usually constraints goals are considered as fixed quantity .But in real life problem ,the constraint goal can not be always exact. So we can consider the constraint goal for less than type constraints at least $b_{j}(s)$ and it may possible to extend to $b_{j}^{0}(s)$ so that the maximum allowable tolerance is $b_{j}^{1}(s)$ so that $b_{j}^{0}(s)=b_{j}(s)+b_{j}^{1}(s)$. This fact seems to take the constraint goal as a intuitionistic fuzzy set and which will be more 
realistic descriptions than others. Then the NLP becomes IFO problem with intuitionistic resources, which can be described as follows

Minimize $f(x)$

$g_{j}(x ; s) \leq \tilde{b}_{j}^{i}(s) \quad j=1,2, \ldots, m$

$x \geq 0 ; s \in[0,1]$

To solve the IFO (3), following warner's (1987) and Angelov (1995) we are presenting a solution procedure for single-objective NSO problem (3) as follows

Step-1: Following warner's approach solve the single objective non-linear programming problem without tolerance in constraints (i.e $g_{j}(x ; s) \leq b_{j}(s)$ ), with tolerance of acceptance in constraints (i.e $\left.g_{j}(x ; s) \leq b_{j}^{0}(s)\right)$ by appropriate non-linear programming technique

Here they are

\section{Sub-problem-1}

Minimize $f(x)$

$$
g_{j}(x ; s) \leq b_{j}(s) \quad j=1,2, \ldots, m
$$

$x \geq 0 ; s \in[0,1]$

\section{Sub-problem-2}

Minimize $f(x)$

$g_{j}(x ; s) \leq b_{j}^{0}(s), \quad j=1,2, \ldots ., m$

$x \geq 0 ; s \in[0,1]$

we may get optimal solutions $x^{*}=x^{1}, f\left(x^{*}\right)=f\left(x^{1}\right)$ and $x^{*}=x^{2}, f\left(x^{*}\right)=f\left(x^{2}\right)$ for subproblem 1 and 2 respectively.

Step-2: From the result of step 1 we now find the lower bound and upper bound of objective functions. If $U_{f(x)}^{\mu}, U_{f(x)}^{v}$ be the upper bounds of truth, indeterminacy, falsity function for the objective respectively and $L_{f(x)}^{\mu}, L_{f(x)}^{\nu}$ be the lower bound of membership and non-membership functions of objective respectively then 


$$
\begin{aligned}
& U_{f(x)}^{\mu}=\max \left\{f\left(x^{1}\right), f\left(x^{2}\right)\right\}, L_{f(x)}^{\mu}=\min \left\{f\left(x^{1}\right), f\left(x^{2}\right)\right\}, \\
& U_{f(x)}^{v}=U_{f(x)}^{\mu}, L_{f(x)}^{v}=L_{f(x)}^{\mu}+\varepsilon_{f(x)} \text { where } 0<\varepsilon_{f(x)}<\left(U_{f(x)}^{\mu}-L_{f(x)}^{\mu}\right)
\end{aligned}
$$

Step-3: In this step we calculate linear membership for membership and non membership functions of objective as follows

$$
\begin{aligned}
& \mu_{f(x)}(f(x))=\left\{\begin{array}{cc}
1 & \text { if } f(x) \leq L_{f(x)}^{\mu} \\
\left.\frac{U_{f(x)}^{\mu}-f(x)}{U_{f(x)}^{\mu}-L_{f(x)}^{\mu}}\right) & \text { if } L_{f(x)}^{\mu} \leq f(x) \leq U_{f(x)}^{\mu} \\
0 & \text { if } f(x) \geq U_{f(x)}^{\mu}
\end{array}\right. \\
& v_{f(x)}(f(x))=\left\{\begin{array}{cc}
0 & \text { if } f(x) \leq L_{f(x)}^{v} \\
\frac{f(x)-L_{f(x)}^{v}}{U_{f(x)}^{v}-L_{f(x)}^{v}} & \text { if } L_{f(x)}^{v} \leq f(x) \leq U_{f(x)}^{v} \\
1 & \text { if } f(x) \geq U_{f(x)}^{v}
\end{array}\right.
\end{aligned}
$$

Step-4: In this step using linear function for membership and non-membership functions, we may calculate membership and non membership function for constraints as follows

$$
\begin{aligned}
& \mu_{g_{j}(x ; s)}\left(g_{j}(x ; s)\right)=\left\{\begin{array}{cc}
1 & \text { if } g_{j}(x ; s) \leq b_{j}(s) \\
\left.\frac{b_{j}^{0}(s)-g_{j}(x ; s)}{b_{j}^{1}(s)}\right) & \text { if } b_{j}(s) \leq g_{j}(x ; s) \leq b_{j}^{0}(s) \\
0 & \text { if } g_{j}(x ; s) \geq b_{j}^{0}(s)
\end{array}\right. \\
& v_{g_{j}(x ; s)}\left(g_{j}(x ; s)\right)=\left\{\begin{array}{cc}
0 & \text { if } g_{j}(x ; s) \leq b_{j}(s)+\varepsilon_{g_{j}(x ; s)} \\
\frac{g_{j}(x ; s)-b_{j}(s)-\varepsilon_{g_{j}(x ; s)}}{b_{j}^{1}(s)-\varepsilon_{g_{j}(x ; s)}} & \text { if } b_{j}(s)+\varepsilon_{g_{j}(x ; s)} \leq g_{j}(x ; s) \leq b_{j}^{0}(s) \\
1 & \text { if } g_{j}(x ; s) \geq b_{j}^{0}(s)
\end{array}\right.
\end{aligned}
$$

where and for $j=1,2, \ldots . ., m \quad 0<\varepsilon_{g_{j}(x ; s)}<b_{j}^{1}(s)$.

$x \geq 0 ; s \in[0,1]$ 
Step-5: Now using Fuzzy and IFO for single objective optimization technique(Singh .et.al [23]) the optimization problem (2) can be formulated as

Maximize $\alpha$

Such that

$\mu_{f(x)}(x) \geq \alpha ; \mu_{g_{j}}(x ; s) \geq \alpha ;$

$$
\alpha \in[0,1]
$$

$x \geq 0 ; s \in[0,1]$

and

Maximize $(\alpha-\beta)$

Suh that

$\mu_{f(x)}(x) \geq \alpha ; \mu_{g_{j}}(x ; s) \geq \alpha ;$

$v_{f(x)}(x) \leq \beta ; v_{g_{j}}(x ; s) \leq \beta$;

$\alpha+\beta \leq 1 ; \alpha \geq \beta$

$$
\alpha, \beta \in[0,1] x \geq 0 ; s \in[0,1]
$$

respectively.Now the above problem (6),(7) can be solved appropriate mathematical programming.

\section{SOLUTION OF SINGLE-OBJECTIVE WELDED BEAM DESIGN(SOWBD) BY FUZZY AND INTUITIONISTIC FUZZY OPTIMIZATION TECHNIQUE}

The parametric Welded beam design problem can be formulated as

Minimize $C(X)$

subject to $\sigma_{i}(X ; s) \leq\left[\sigma_{i}(s)\right], i=1,2, \ldots . ., m$

$X_{j} \in R^{d}, \quad j=1,2, \ldots ., n$

$X>0 ; s \in[0,1]$ 
where $C(X)$ represents cost function, $\sigma_{i}(X)$ is the behavioural constraints and $\left[\sigma_{i}(X)\right]$ denotes the maximum allowable value , ' $m$ ' and ' $n$ ' are the number of constraints and design variables respectively. A given set of discrete value is expressed by $R^{d}$ and in this paper objective function is taken as

$C(X)=\sum_{t=1}^{T} c_{t} \prod_{n=1}^{m} x_{n}^{t n}$

and constraint are chosen to be stress of structures as follows

$\sigma_{i}(X ; s) \lesssim \sigma_{i}(s)$ with allowable tolerance $\sigma_{i}^{0}(s)$ for $i=1,2, \ldots ., m$

And deflection of the structure as follows

$\delta(X ; s) \lesssim \delta_{\max }(s)$ with allowable tolerance $\delta_{\max }^{0}(s)$

Where $c_{t}$ is the cost coefficient of $\mathrm{t}^{\text {th }}$ side and $x_{n}$ is the $n^{\text {th }}$ design variable

respectively, $m$ is the number of structural element, $\sigma_{i}$ and $\sigma_{i}^{0}(s) \delta_{\max }^{0}(s)$ are the $i^{\text {th }}$ stress, allowable stress and allowable deflection respectively.

To solve the SOWBP (8), step 1 of 4 is used and we will get optimum solutions of two sub problem as $X^{1}$ and $X^{2}$.After that according to step 2 we find upper and lower bound of membership function of objective function as $U_{C(X)}^{\mu}, U_{C(X)}^{v}$ and $L_{C(X)}^{\mu}, L_{C(X)}^{\nu} \quad$ where $U_{C(X)}^{\mu}=\max \left\{C\left(X^{1}\right), C\left(X^{2}\right)\right\}, L_{C(X)}^{\mu}=\min \left\{C\left(X^{1}\right), C\left(X^{2}\right)\right\}$, $U_{C(X)}^{v}=U_{C(X)}^{\mu}, L_{C(X)}^{\nu}=L_{C(X)}^{\mu}+\varepsilon_{C(X)}$ where $0<\varepsilon_{C(X)}<\left(U_{C(X)}^{\mu}-L_{C(X)}^{\mu}\right)$

Let the linear membership function for objective be $\mu_{C(X)}(C(X))=\left\{\begin{array}{cc}1 & \text { if } C(X) \leq L_{C(X)}^{\mu} \\ \left.\frac{U_{C(X)}^{\mu}-C(X)}{U_{C(X)}^{\mu}-L_{C(X)}^{\mu}}\right) & \text { if } L_{C(X)}^{\mu} \leq C(X) \leq U_{C(X)}^{\mu} \\ 0 & \text { if } C(X) \geq U_{C(X)}^{\mu}\end{array}\right.$ 


$$
v_{C(X)}(C(X))=\left\{\begin{array}{cl}
0 & \text { if } C(X) \leq L_{C(X)}^{\mu}+\varepsilon_{C(X)} \\
\left(\frac{C(X)-\left(L_{C(X)}^{\mu}+\varepsilon_{C(X)}\right)}{\left.U_{C(X)}^{\mu}-L_{C(X)}^{\mu}-\varepsilon_{C(X)}\right)}\right) & \text { if } L_{C(X)}^{\mu}+\varepsilon_{C(X)} \leq C(X) \leq U_{C(X)}^{\mu} \\
1 & \text { if } C(X) \geq U_{C(X)}^{\mu}
\end{array}\right.
$$

and constraints be

$$
\begin{aligned}
& \mu_{g_{j}(X ; s)}\left(g_{j}(X ; s)\right)=\left\{\begin{array}{cc}
1 & \text { if } g_{j}(X ; s) \leq b_{j}(s) \\
\left(\frac{b_{j}^{0}(s)-g_{j}(X ; s)}{b_{j}^{1}(s)}\right) & \text { if } b_{j}(s) \leq g_{j}(X ; s) \leq b_{j}^{0}(s) \\
0 & \text { if } g_{j}(X ; s) \geq b_{j}^{0}(s)
\end{array}\right. \\
& v_{g_{j}(X ; s)}\left(g_{j}(X ; s)\right)=\left\{\begin{array}{cc}
0 & \text { if } g_{j}(X ; s) \leq b_{j}(s)+\varepsilon_{g_{j}(x ; s)} \\
\frac{g_{j}(X ; s)-b_{j}(s)-\varepsilon_{g_{j}(X ; s)}}{b_{j}^{0}(s)-\varepsilon_{g_{j}(X ; s)}} & \text { if } b_{j}(s)+\varepsilon_{g_{j}(X ; s)} \leq g_{j}(X ; s) \leq b_{j}^{0}(s) \\
1 & \text { if } g_{j}(X ; s) \geq b_{j}^{0}(s)
\end{array}\right.
\end{aligned}
$$

where and for $g_{i}(X ; s)=\sigma_{i}(X ; s)$ or $\delta_{i}(X ; s)$ or $\tau_{i}(X ; s), 0<\varepsilon_{g_{j}(X ; s)}<b_{j}^{0}(s)$

then parametric fuzzy and intuitionistic fuzzy optimization problem can be formulated as

Maximize $\alpha$

such that

$$
\begin{aligned}
& \mu_{C(X ; s)}(C(X ; s)) \geq \alpha ; \mu_{g_{j}(X ; s)}\left(g_{j}(X ; s)\right) \geq \alpha ; \\
& \qquad g_{i}(X ; s) \leq\left[g_{i}(s)\right] ; \alpha \in[0,1] X \geq 0 ; s \in[0,1] \\
& g_{i}(X ; s)=\sigma_{i}(X ; s) \text { or } \delta_{i}(X ; s) \text { or } \tau_{i}(X ; s), 0<\varepsilon_{g_{j}(X ; s)}<b_{j}^{1}(s) \\
& \text { and }
\end{aligned}
$$

Maximize $(\alpha-\beta)$

such that 


$$
\begin{aligned}
& \mu_{C(X ; s)}(C(X ; s)) \geq \alpha ; \mu_{g_{j}(X ; s)}\left(g_{j}(X ; s)\right) \geq \alpha ; \\
& v_{C(X ; s)}(C(X ; s)) \leq \beta ; v_{g_{j}(X ; s)}\left(g_{j}(X ; s)\right) \leq \beta \\
& \quad g_{i}(X ; s) \leq\left[g_{i}(s)\right] ; \alpha+\beta \leq 1 ; \alpha \geq \beta ; \alpha, \beta \in[0,1] X \geq 0 ; s \in[0,1] \\
& g_{i}(X ; s)=\sigma_{i}(X ; s) \text { or } \delta_{i}(X ; s) \text { or } \tau_{i}(X ; s), 0<\varepsilon_{g_{j}(X ; s)}<b_{j}^{1}(s)
\end{aligned}
$$

respectively. Now the above problems can be solved by appropriate mathematical programming.

\section{NUMERICAL ILLUSTRATION}

A welded beam (Ragsdell and Philips 1976,Fig. 2) has to be designed at minimum cost whose constraints are shear stress in weld $(\tau)$, bending stress in the beam $(\sigma)$ ,buckling load on the bar $(P)$, and deflection of the beam $(\delta)$.The design variables are $\left[\begin{array}{l}x_{1} \\ x_{2} \\ x_{3} \\ x_{4}\end{array}\right]=\left[\begin{array}{l}h \\ l \\ t \\ b\end{array}\right]$ where $h$ is the the weld size, $l$ is the length of the weld, $t$ is the depth of the welded beam, $b$ is the width of the welded beam.

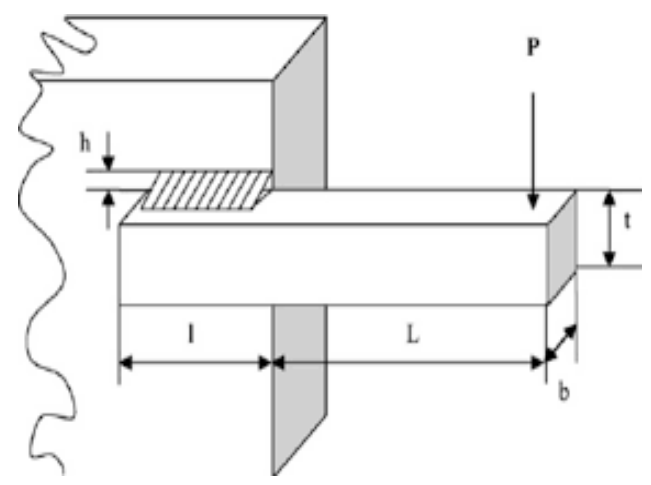

Fig.2. Design of the welded beam

\section{Cost Function}

The performance index appropriate to this design is the cost of weld assembly. The major cost components of such an assembly are (i) set up labour cost, (ii) welding labour cost, (iii) material cost. 
Optimization of Welded Beam with Imprecise Load and Stress by Parameterized.. 595

$C(X) \equiv C_{0}+C_{1}+C_{2}$ where, $f(X)=$ cost function; $C_{0}=$ set up cost; $C_{1}=$ welding labour cost; $C_{2}=$ material cost;

Set up cost $C_{0}$ : The company has chosen to make this component a weldment, because of the existence of a welding assembly line. Furthermore, assume that fixtures for set up and holding of the bar during welding are readily available. The cost $C_{0}$ can therefore be ignored in this particular total cost model.

Welding labour cost $C_{1}$ : Assume that the welding will be done by machine at a total cost of $\$ 10 / \mathrm{hr}$ (including operating and maintenance expense). Furthermore suppose that the machine can lay down a cubic inch of weld in $6 \mathrm{~min}$. The labour cost is then

$$
C_{1}=\left(10 \frac{\$}{h r}\right)\left(\frac{1}{60} \frac{\$}{\min }\right)\left(6 \frac{\min }{i n^{3}}\right) V_{w}=1\left(\frac{\$}{i n^{3}}\right) V_{w} . \text { Where } V_{w}=\text { weld volume, } \mathrm{in}^{3}
$$

Material cost $C_{2}: C_{2}=C_{3} V_{w}+C_{4} V_{B}$. Where $C_{3}=$ cost per volume per weld material. $\$ / \mathrm{in}^{3}=(0.37)(0.283) \quad ; \quad C_{4}=$ cost per volume of bar stock. $\$ / \mathrm{in}^{3}$ $=(0.37)(0.283) ; V_{B}=$ volume of bar, in ${ }^{3}$.From geometry $V_{w}=h^{2} l ;$ volume of the weld material $\left(\mathrm{in}^{3}\right) \quad V_{\text {weld }}=x_{1}^{2} x_{2}$ and $V_{B}=t b(L+l)$; volume of bar $\left(\mathrm{in}^{3}\right)$ $V_{b a r}=x_{3} x_{4}\left(L+x_{2}\right)$ Therefore cost function become

$$
C(X)=h^{2} l+C_{3} h^{2} l+C_{4} t b(L+l)=1.10471 x_{1}^{2} x_{2}+0.04811 x_{3} x_{4}\left(14.0+x_{2}\right)
$$

\section{Engineering Relationship}

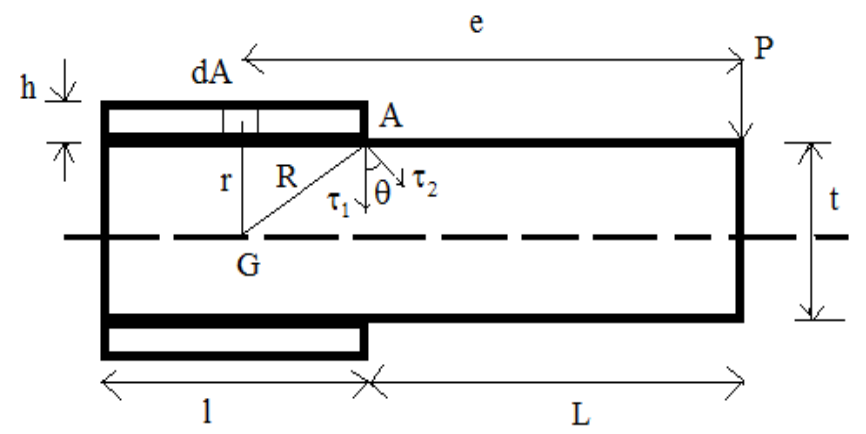

Fig 3. Shear stresses in the weld group.

\section{Maximum shear stress in weld group:}

To complete the model it is necessary to define important stress states 
Direct or primary shear stress $\tau_{1}=\frac{\text { Load }}{\text { Throat area }}=\frac{P}{A}=\frac{P}{\sqrt{2} h l}=\frac{P}{\sqrt{2} x_{1} x_{2}}$

Since the shear stress produced due to turning moment $M=P . e$ at any section is proportional to its radial distance from centre of gravity of the joint ' $G$ ', therefore stress due to $M$ is proportional to $R$ and is in a direction at right angles to $R$. In other words $\frac{\tau_{2}}{R}=\frac{\tau}{r}=$ constant. Therefore $R=\sqrt{\left(\frac{l}{2}\right)^{2}+\left(\frac{h+t}{2}\right)^{2}}=\sqrt{\frac{x_{2}^{2}}{4}+\frac{\left(x_{1}+x_{3}\right)^{2}}{4}}$

Where, $\tau_{2}$ is the shear stress at the maximum distance $R$ and $\tau$ is the shear stress at any distance $r$. Consider a small section of the weld having area $d A$ at a distance $r$ from ' $G$ '. Therefore shear force on this small section $=\tau \times d A$ and turning moment of the shear force about centre of gravity $d M=\tau \times d A \times r=\frac{\tau_{2}}{R} \times d A \times r^{2}$. Therefore total turning moment over the whole weld area $M=\frac{\tau_{2}}{R} \int d A \times r^{2}=\frac{\tau_{2}}{R} J$. where $J=$ polar moment of inertia of the weld group about centre of gravity. Therefore shear stress due to the turning moment i.e. secondary shear stress, $\tau_{2}=\frac{M R}{J}$. In order to find the resultant stress, the primary and secondary shear stresses are combined vectorially. Therefore the maximum resultant shear stress that will be produced at the weld group, $\tau=\sqrt{\tau_{1}^{2}+\tau_{2}^{2}+2 \tau_{1} \tau_{2} \cos \theta}$, where, $\theta=$ Angle between $\tau_{1}$ and $\tau_{2}$.As $\cos \theta=\frac{l / 2}{R}=\frac{x_{2}}{2 R} ; \tau=\sqrt{\tau_{1}^{2}+\tau_{2}^{2}+2 \tau_{1} \tau_{2} \frac{x_{2}}{2 R}}$.

Now the polar moment of inertia of the throat area $(A)$ about the centre of gravity is obtained by parallel axis theorem,

$$
J=2\left[I_{x x}+A+x^{2}\right]=2\left[\frac{A \times l^{2}}{12}+A \times x^{2}\right]=2 A\left(\frac{l^{2}}{12}+x^{2}\right)=2\left\{\sqrt{2} x_{1} x_{2}\left[\frac{x_{2}^{2}}{12}+\frac{\left(x_{1}+x_{3}\right)^{2}}{2}\right]\right\}
$$

Where, $A=$ throat area $=\sqrt{2} x_{1} x_{2}, l=$ Length of the weld, $x=$ Perpendicular distance between two parallel axes $=\frac{t}{2}+\frac{h}{2}=\frac{x_{1}+x_{3}}{2}$.

\section{Maximum bending stress in beam:}


Now Maximum bending moment $=P L$, Maximum bending stress $=\frac{T}{Z}$, where $T=P L$

$Z=$ section modulus $=\frac{I}{y} ; I=$ moment of inertia $=\frac{b t^{3}}{12} ; y=$ distance of extreme fibre from centre of gravity of cross section $=\frac{t}{2}$; Therefore $Z=\frac{b t^{2}}{6}$. So bar bending stress $\sigma(x)=\frac{T}{Z}=\frac{6 P L}{b t^{2}}=\frac{6 P L}{x_{4} x_{3}^{2}}$

\section{Maximum deflection in beam:}

Maximum deflection at cantilever tip $=\frac{P L^{3}}{3 E I}=\frac{P L^{3}}{3 E \frac{b t^{3}}{12}}=\frac{4 P L^{3}}{E b t^{3}}$

\section{Buckling load of beam:}

buckling load can be approximated by $P_{C}(x)=\frac{4.013 \sqrt{E I C}}{l^{2}}\left(1-\frac{a}{l} \sqrt{\frac{E l}{C}}\right)$

where, $I=$ moment of inertia $=\frac{b t^{3}}{12} ;$ torsional rigidity $C=G J=\frac{1}{3} t b^{3} G ; l=L ; a=\frac{t}{2}$;

$$
=\frac{4.013 \sqrt{E \frac{t^{2} b^{6}}{36}}}{L^{2}}\left(1-\frac{t}{2 L} \sqrt{\frac{E}{4 G}}\right)=\frac{4.013 \sqrt{E G x_{3}^{6} x_{4}^{6} / 36}}{L^{2}}\left(1-\frac{x_{3}}{2 L} \sqrt{\frac{E}{4 G}}\right) ;
$$

The single-objective optimization problem can be stated as follows

Minimize $C(X) \equiv 1.10471 x_{1}^{2} x_{2}+0.04811\left(14+x_{2}\right) x_{3} x_{4}$

Such that

$$
\begin{aligned}
& g_{1}(x) \equiv \tau(x)-\tau_{\max } \leq 0 ; \\
& g_{2}(x) \equiv \sigma(x)-\sigma_{\max } \leq 0 ; \\
& g_{3}(x) \equiv x_{1}-x_{4} \leq 0 ;
\end{aligned}
$$


$g_{4}(x) \equiv 0.10471 x_{1}^{2} x_{2}+0.04811 x_{3} x_{4}\left(14+x_{2}\right)-5 \leq 0$

$g_{5}(x) \equiv 0.125-x_{1} \leq 0$

$g_{6}(x) \equiv \delta(x)-\delta_{\max } \leq 0$

$g_{7}(x) \equiv P-P_{C}(x) \leq 0$

$0.1 \leq x_{1}, x_{4} \leq 2.0$

$0.1 \leq x_{2}, x_{3} \leq 2.0$

where $\quad \tau(x)=\sqrt{\tau_{1}^{2}+2 \tau_{1} \tau_{2} \frac{x_{2}}{2 R}+\tau_{2}^{2}} \quad ; \quad \tau_{1}=\frac{P}{\sqrt{2} x_{1} x_{2}} ; \tau_{2}=\frac{M R}{J} ; M=P\left(L+\frac{x_{2}}{2}\right)$;

$R=\sqrt{\frac{x_{2}^{2}}{4}+\left(\frac{x_{1}+x_{3}}{2}\right)^{2}} ; J=\left\{\frac{x_{1} x_{2}}{\sqrt{2}}\left[\frac{x_{2}^{2}}{12}+\left(\frac{x_{1}+x_{3}}{2}\right)^{2}\right]\right\} ; \sigma(x)=\frac{6 P L}{x_{4} x_{3}^{2}} ; \delta(x)=\frac{4 P L^{3}}{E x_{4} x_{3}^{2}} ;$

$P_{C}(x)=\frac{4.013 \sqrt{E G x_{3}^{6} x_{4}^{6} / 36}}{L^{2}}\left(1-\frac{x_{3}}{2 L} \sqrt{\frac{E}{4 G}}\right) ; \quad P=$ Force on beam $; L=$ Beam length beyond weld; $x_{1}=$ Height of the welded beam; $x_{2}=$ Length of the welded beam; $x_{3}=$ Depth of the welded beam; $x_{4}=$ Width of the welded beam; $\tau(x)=$ Design shear stress; $\sigma(x)=$ Design normal stress for beam material; $M=$ Moment of $P$ about the centre of gravity of the weld,$J=$ Polar moment of inertia of weld group; $G=$ Shearing modulus of Beam Material; $E=$ Young modulus; $\tau_{\max }=$ Design Stress of the weld; $\sigma_{\max }=$ Design normal stress for the beam material; $\delta_{\max }=$ Maximum deflection; $\tau_{1}=$ Primary stress on weld throat. $\tau_{2}=$ Secondary torsional stress on weld. Input data are given in table 1 and 5.

Table 1: Input data for crisp model (11)

\begin{tabular}{|c|c|c|c|c|c|c|}
\hline $\begin{array}{c}\text { Applied load } \\
P \\
(l b)\end{array}$ & $\begin{array}{c}\text { Beam } \\
\text { length } \\
\text { beyond } \\
\text { weld } L \\
\text { (in) }\end{array}$ & $\begin{array}{c}\text { Young } \\
\text { Modulu } \\
\text { s } \\
\mathrm{E} \\
(p s i)\end{array}$ & $\begin{array}{c}\text { Value of } \\
\quad G \\
(p s i)\end{array}$ & $\begin{array}{l}\text { Maximum } \\
\text { allowable } \\
\text { shear } \\
\text { stress } \tau_{\max } \\
\quad(p s i)\end{array}$ & $\begin{array}{c}\text { Maximum } \\
\text { allowable } \\
\text { deflection } \\
\delta_{\max } \\
(\text { in })\end{array}$ & $\begin{array}{c}\text { Maximum } \\
\text { allowable } \\
\text { normal stress } \\
\sigma_{\max } \\
(p s i)\end{array}$ \\
\hline $\begin{array}{l}6000 \\
\equiv(5900,6000,6\end{array}$ & $100 ; w_{p}^{14}$ & $3 \times 10^{6}$ & $12 \times 10^{6}$ & $\begin{array}{l}13550 \\
\equiv\left(135400,13550,13560 ; w_{\tau}\right) \\
\\
\text { Maximum } \\
\text { allowable } \\
\text { value }\end{array}$ & $\begin{array}{l}0.2 \tilde{5} \\
\equiv\left(0.24,0.25,0.26 ; w_{\delta}\right) \\
\text { Maximum } \\
\text { allowable } \\
\quad \text { value }\end{array}$ & $\begin{array}{l}3000 \\
\equiv\left(2900,3000,3100 ; w_{\sigma}\right) \\
\text { Maximum } \\
\text { allowable } \\
\text { value }\end{array}$ \\
\hline
\end{tabular}


Optimization of Welded Beam with Imprecise Load and Stress by Parameterized.. 599

\begin{tabular}{|l|l|l|l|l|l|}
\hline & & & $\begin{array}{l}1360 \tilde{0} \\
\equiv\left(13500,13600,13700 ; w_{\tau}^{1}\right)\end{array}$ & $\begin{array}{l}0.2 \tilde{6} \\
\equiv\left(0.25,0.26,0.27 ; w_{\delta}^{1}\right)\end{array}$ & $\begin{array}{l}310 \tilde{0} \\
\equiv\left(3000,3100,3200 ; w_{\sigma}^{1}\right)\end{array}$ \\
\hline
\end{tabular}

where $w_{p}, w_{\sigma}, w_{\delta}, w_{\tau}$ and $w_{p}, w_{\sigma}^{1}, w_{\delta}^{1}, w_{\tau}^{1}$ are degree of acceptance or aspiration level and maximum degree of acceptance or aspiration level of applied load, normal stress ,deflection and allowable shear stress respectively.

Now parameterized value of interval valued function can be calculated as

$$
\begin{aligned}
& \hat{P}=\left(\left(\frac{\left(2 w_{p}-1\right) 5900+6000}{2 w_{p}}\right)^{1-s}\left(\frac{\left(2 w_{p}-1\right) 6100+6000}{2 w_{p}}\right)^{s}\right) ; \\
& \hat{\tau}^{\max }=\left(\left(\frac{\left(2 w_{\tau}-1\right) 13400+13500}{2 w_{\tau}}\right)^{1-s}\left(\frac{\left(2 w_{\tau}-1\right) 13600+13500}{2 w_{\tau}}\right)^{s}\right) ;
\end{aligned}
$$

Allowable value of $\hat{\tau}^{\max }$

$$
\begin{aligned}
& \hat{\tau}_{1}^{\max }=\left(\left(\frac{\left(2 w_{\tau_{1}}-1\right) 13500+13600}{2 w_{\tau_{1}}}\right)^{1-s}\left(\frac{\left(2 w_{\tau_{1}}-1\right) 13700+13600}{2 w_{\tau_{1}}}\right)^{s}\right) ; \\
& \hat{\delta}^{\max }=\left(\left(\frac{\left(2 w_{\delta}-1\right) 0.24+0.25}{2 w_{\delta}}\right)^{1-s}\left(\frac{\left(2 w_{\delta}-1\right) 0.26+0.25}{2 w_{\delta}}\right)^{s}\right) ;
\end{aligned}
$$

Allowable value of $\hat{\delta}^{\max }$

$$
\begin{aligned}
& \hat{\delta}_{1}^{\max }=\left(\left(\frac{\left(2 w_{\delta_{1}}-1\right) 0.25+0.26}{2 w_{\delta_{1}}}\right)^{1-s}\left(\frac{\left(2 w_{\delta_{1}}-1\right) 0.27+0.26}{2 w_{\delta_{1}}}\right)^{s}\right) ; \\
& \hat{\sigma}^{\max }=\left(\left(\frac{\left(2 w_{\sigma}-1\right) 2900+3000}{2 w_{\sigma}}\right)^{1-s}\left(\frac{\left(2 w_{\sigma}-1\right) 3100+3000}{2 w_{\sigma}}\right)^{s}\right) ;
\end{aligned}
$$

Allowable value of $\hat{\sigma}^{\max }$

$$
\hat{\sigma}_{1}^{\max }=\left(\left(\frac{\left(2 w_{\sigma_{1}}-1\right) 3000+3100}{2 w_{\sigma_{1}}}\right)^{1-s}\left(\frac{\left(2 w_{\sigma_{1}}-1\right) 3200+3100}{2 w_{\sigma_{1}}}\right)^{s}\right)
$$


Table. 2. The Upper and lower value of objective for different values of $w$ pessimistic value of $s$

\begin{tabular}{|c|c|c|c|}
\hline \multicolumn{4}{|c|}{ The pessimistic value of $s=0.2$} \\
\hline $\begin{array}{c}\text { Aspiration level } \\
w_{p}=w_{\sigma}=w_{\delta}=w_{\tau} \\
=w_{p}=w_{\sigma}^{1}=w_{\delta}^{1}=w_{\tau}^{1}=w\end{array}$ & $w=0.3$ & $w=0.5$ & $w=0.7$ \\
\hline $\begin{array}{l}\text { Upper and lower value of } \\
\text { objective }\end{array}$ & $\begin{array}{l}L_{C(X)}^{\mu}=0.1264788, \\
U_{C(X)}^{\mu}=0.1433088\end{array}$ & $\begin{array}{l}L_{C(X)}^{\mu}=0.1258191, \\
U_{C(X)}^{\mu}=0.1426818\end{array}$ & $\begin{array}{l}L_{C(X)}^{\mu}=0.1255331, \\
U_{C(X)}^{\mu}=0.1424090\end{array}$ \\
\hline
\end{tabular}

Table.3 The Upper and lower value of objective for different values of $w$,moderate value of value of $s$

\begin{tabular}{|l|l|l|l|}
\hline \multicolumn{1}{|c|}{ The pessimistic value of $\mathrm{s}=0.5$} \\
$\begin{array}{l}\text { Aspiration level } \\
w_{p}=w_{\sigma}=w_{\delta}=w_{\tau} \\
=w_{p}=w_{\sigma}^{1}=w_{\delta}^{1}=w_{\tau}^{1}=w\end{array}$ & $w=0.3$ & $w=0.5$ & $w=0.7$ \\
\hline $\begin{array}{l}\text { Upper and lower value } \\
\text { of objective }\end{array}$ & $\begin{array}{l}L_{C(X)}^{\mu}=0.1426734, \\
U_{C(X)}^{\mu}=0.1438970\end{array}$ & $\begin{array}{l}L_{C(X)}^{\mu}=0.1426818, \\
U_{C(X)}^{\mu}=0.1439054\end{array}$ & $\begin{array}{l}L_{C(X)}^{\mu}=0.1426802, \\
U_{C(X)}^{\mu}=0.1439054\end{array}$ \\
\hline
\end{tabular}

Table.4 The Upper and lower value of objective for different values of $\quad w$ optimistic value of $s$

\begin{tabular}{|c|l|l|l|}
\hline \multicolumn{3}{|c|}{ The pessimistic value of $\mathrm{s}=0.8$} \\
\hline $\begin{array}{c}\text { Aspiration level } \\
w_{p}=w_{\sigma}=w_{\delta}=w_{\tau}\end{array}$ & $w=0.3$ & $w=0.5$ & $w=0.7$ \\
$=w_{p}=w_{\sigma}^{1}=w_{\delta}^{1}=w_{\tau}^{1}=w$ & & & \\
\hline
\end{tabular}




\begin{tabular}{|l|l|l|l|}
\hline $\begin{array}{l}\text { Upper and lower value } \\
\text { of objective }\end{array}$ & $\begin{array}{l}L_{C(X)}^{\mu}=0.1420414, \\
U_{C(X)}^{\mu}=0.1432617\end{array}$ & $\begin{array}{l}L_{C(X)}^{\mu}=0.1426818, \\
U_{c(X)}^{\mu}=0.1439054\end{array}$ & $\begin{array}{l}L_{C(X)}^{\mu}=0.1429521, \\
U_{c(X)}^{\mu}=0.1441772\end{array}$ \\
\hline
\end{tabular}

Again when input parameters are as like as table 5

Table 5: Input data for crisp model (9)

\begin{tabular}{|c|c|c|c|c|c|c|}
\hline $\begin{array}{l}\text { Applied } \\
\text { load } P \\
\quad(l b)\end{array}$ & $\begin{array}{c}\text { Beam } \\
\text { lengt } \\
\mathrm{h} \\
\text { beyon } \\
\mathrm{d} \\
\text { weld } \\
L \\
(\mathrm{in})\end{array}$ & $\begin{array}{l}\text { Young } \\
\text { Modul } \\
\text { us } \\
\text { E } \\
(p s i)\end{array}$ & $\begin{array}{l}\text { Value } \\
\text { of } \\
G \\
(p s i)\end{array}$ & $\begin{array}{l}\text { Maximum } \\
\text { allowable shear } \\
\text { stress } \tau_{\max } \\
\quad(p s i)\end{array}$ & $\begin{array}{c}\text { Maximum } \\
\text { allowable } \\
\text { deflection } \\
\delta_{\max } \\
(\text { in })\end{array}$ & $\begin{array}{c}\text { Maximum } \\
\text { allowable } \\
\text { normal stress } \\
\sigma_{\max } \\
(p s i)\end{array}$ \\
\hline $\begin{array}{l}6000 \tilde{0} \\
\equiv\left(\begin{array}{l}\left(5900,6000,6100 ; w_{p}\right. \\
\left(5800,6000,620 ; ; \eta_{p}\right.\end{array}\right)\end{array}$ & 14 & $3 \times 10^{6}$ & $12 \times 10^{6}$ & $\begin{array}{l}13550 \\
\equiv\left(\begin{array}{l}\left(13540,13550,13560 ; w_{\tau}\right. \\
\left(13530,13550,13570 ; \eta_{\tau}\right.\end{array}\right. \\
\text { Maximum } \\
\text { allowable value } \\
1360000 \\
\equiv\left(\begin{array}{l}\left(13500,13600,13700 ; w_{\tau}^{1}\right) \\
\left(13400,13600,13800 ; \eta_{\tau}^{1}\right)\end{array}\right)\end{array}$ & $\begin{array}{l}0.2 \tilde{5} \\
=\left(\begin{array}{l}\left(0.24,0.25,0.26 ; w_{\delta}\right) \\
\left(0.23,0.25,0.27 ; \eta_{\delta}\right)\end{array}\right) \\
\begin{array}{l}\text { Maximum } \\
\text { allowable } \\
\text { value }\end{array} \\
0.2 \tilde{6} \\
\equiv\left(\begin{array}{l}\left(0.25,0.26,0.27 ; w_{\delta}^{1}\right. \\
\left(0.24,0.26,0.28 ; \eta_{\delta}^{1}\right.\end{array}\right)\end{array}$ & 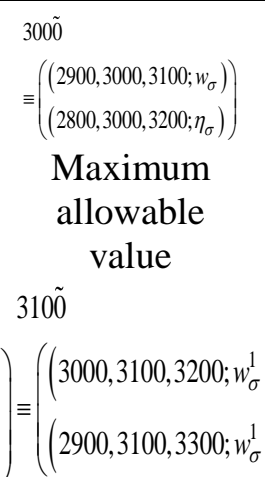 \\
\hline
\end{tabular}

where $w_{p}, w_{\sigma}, w_{\delta}, w_{\tau}$ and $w_{p}, w_{\sigma}^{1}, w_{\delta}^{1}, w_{\tau}^{1}$ are degree of acceptance or aspiration level and maximum degree of acceptance or aspiration level of applied load, normal stress ,deflection and allowable shear stress respectively. and $\eta_{p}, \eta_{\sigma}, \eta_{\delta}, \eta_{\tau}$ and $\eta_{p}, \eta_{\sigma}^{1}, \eta_{\delta}^{1}, \eta_{\tau}^{1}$ are degree of rejection or desperation level and maximum degree of rejection or desperation level of applied load, normal stress ,deflection and allowable shear stress respectively.

Now parameterized value of interval valued function can be calculated as

$$
\begin{aligned}
& \hat{P}=\left(\left(5950+\frac{25}{w_{p}}+\frac{50}{\eta_{p}}\right)^{1-s}\left(6050+\frac{25}{w_{p}}+\frac{50}{\eta_{p}}\right)^{s}\right) \\
& \hat{\tau}^{\max }=\left(\left(13545+\frac{2.5}{w_{\tau}}+\frac{5}{\eta_{\tau}}\right)^{1-s}\left(13555+\frac{2.5}{w_{\tau}}+\frac{50}{\eta_{\tau}}\right)^{s}\right) ;
\end{aligned}
$$


Allowable value of $\hat{\tau}^{\max }$

$$
\begin{aligned}
& \hat{\tau}_{1}^{\max }=\left(\left(13550+\frac{25}{w_{\tau_{1}}}+\frac{50}{\eta_{\tau_{1}}}\right)^{1-s}\left(13650+\frac{25}{w_{\tau_{1}}}+\frac{50}{\eta_{\tau_{1}}}\right)^{s}\right) \\
& \hat{\delta}^{\max }=\left(\left(0.145+\frac{.0025}{w_{\delta}}+\frac{.005}{\eta_{\delta}}\right)^{1-s}\left(0.255+\frac{.0025}{w_{\delta}}+\frac{.005}{\eta_{\delta}}\right)^{s}\right) ;
\end{aligned}
$$

Allowable value of $\hat{\delta}^{\max }$

$$
\begin{aligned}
& \hat{\delta}_{1}^{\max }=\left(\left(0.255+\frac{.0025}{w_{\delta_{1}}}+\frac{.005}{\eta_{\delta_{1}}}\right)^{1-s}\left(0.265+\frac{.0025}{w_{\delta_{1}}}+\frac{.005}{\eta_{\delta_{1}}}\right)^{s}\right) \\
& \hat{\sigma}^{\max }=\left(\left(2950+\frac{25}{w_{\sigma}}+\frac{50}{\eta_{\sigma}}\right)^{1-s}\left(3050+\frac{25}{w_{\sigma}}+\frac{50}{\eta_{\sigma}}\right)^{s}\right)
\end{aligned}
$$

Allowable value of $\hat{\sigma}^{\max }$

$$
\hat{\sigma}_{1}^{\max }=\left(\left(3050+\frac{25}{w_{\sigma_{1}}}+\frac{50}{\eta_{\sigma_{1}}}\right)^{1-s}\left(3150+\frac{25}{w_{\sigma_{1}}}+\frac{50}{\eta_{\sigma_{1}}}\right)^{s}\right)
$$

Table. 6 The upper and lower value of objective for different values of $\quad w, \eta$ pessimistic value of $s$

\begin{tabular}{|l|l|l|l|}
\hline \multicolumn{3}{|c|}{ The pessimistic value of $\mathrm{s}=0.2$} \\
$\begin{array}{l}\text { Aspiration level } \\
w_{p}=w_{\sigma}=w_{\delta}=w_{\tau}\end{array}$ & $w=0.3, \eta=0.6$ & $w=0.5, \eta=0.5$ & $w=0.7, \eta=0.2$ \\
$=w_{\sigma}^{1}=w_{\delta}^{1}=w_{\tau}^{1}=w$ & & & \\
and & & & \\
Desperation level & & & \\
$\eta_{p}=\eta_{\sigma}=\eta_{\delta}=\eta_{\tau}$ & & & \\
$=\eta_{\sigma}^{1}=\eta_{\delta}^{1}=\eta_{\tau}^{1}=\eta$ & & & \\
\hline
\end{tabular}




\begin{tabular}{|l|l|l|l|}
\hline $\begin{array}{l}\text { Upper and lower value } \\
\text { of objective }\end{array}$ & $\begin{array}{l}L_{C(X)}^{\mu}=0.1448289, \\
U_{C(X)}^{\mu}=0.1466027\end{array}$ & $L_{C(X)}^{\mu}=0.1445683$, & $L_{C(X)}^{\mu}=0.1466777$, \\
& & & \\
& & & \\
\hline
\end{tabular}

Table.7 The upper and lower value of objective for different values of $\quad w, \eta$, moderate value of $s$

\begin{tabular}{|l|l|l|l|}
\hline \multicolumn{4}{|c|}{ The moderate value of $\mathrm{s}=0.5$} \\
\hline $\begin{array}{c}\text { Aspiration level } \\
w_{p}=w_{\sigma}=w_{\delta}=w_{\tau} \\
=w_{\sigma}^{1}=w_{\delta}^{1}=w_{\tau}^{1}=w \\
\text { and }\end{array}$ & $w=0.3, \eta=0.6$ & $w=0.5, \eta=0.5$ & $w=0.7, \eta=0.2$ \\
$\begin{array}{l}\text { Desperation level } \\
\eta_{p}=\eta_{\sigma}=\eta_{\delta}=\eta_{\tau} \\
=\eta_{\sigma}^{1}=\eta_{\delta}^{1}=\eta_{\tau}^{1}=\eta\end{array}$ & & & \\
\hline $\begin{array}{l}\text { Upper and lower } \\
\text { value of objective }\end{array}$ & $L_{c(x)}^{\mu}=0.1452953$, & $L_{c(X)}^{\mu}=0.1450354$, & \\
\hline
\end{tabular}

Table.8 The upper and lower value of objective for different values of $\quad w, \eta$ optimistic value of $s$

\begin{tabular}{|l|l|l|l|}
\hline \multicolumn{3}{|c|}{ The optimistic value of $\mathrm{s}=0.8$} \\
\hline \multicolumn{1}{|c|}{ Aspiration level } & $w=0.3, \eta=0.6$ & $w=0.5, \eta=0.5$ & $w=0.7, \eta=0.2$ \\
$w_{p}=w_{\sigma}=w_{\delta}=w_{\tau}$ & & & \\
$=w_{\sigma}^{1}=w_{\delta}^{1}=w_{\tau}^{1}=w$ & & & \\
and & & & \\
Desperation level & & & \\
\hline
\end{tabular}




\begin{tabular}{|c|c|c|c|}
\hline $\begin{array}{l}\eta_{p}=\eta_{\sigma}=\eta_{\delta}=\eta_{\tau} \\
=\eta_{\sigma}^{1}=\eta_{\delta}^{1}=\eta_{\tau}^{1}=\eta\end{array}$ & & & \\
\hline $\begin{array}{l}\text { Upper and lower } \\
\text { value of objective }\end{array}$ & $\begin{array}{l}L_{C(X)}^{\mu}=0.1457635, \\
U_{C(X)}^{\mu}=0.1470916\end{array}$ & $\begin{array}{l}L_{C(X)}^{\mu}=0.1455044, \\
U_{C(X)}^{\mu}=0.1467933\end{array}$ & $\begin{array}{l}L_{C(X)}^{\mu}=0.1476010, \\
U_{C(X)}^{\mu}=0.1492130\end{array}$ \\
\hline
\end{tabular}

Now using membership and non membership function as mentioned in section 5 fuzzy and intuitionistic optimization problem can be formulated as similar as (9) and (10) and solving these for different values of $s, w, \tau$ can be obtained as follows.

Table.9 The optimum values of design variables for different values of $s, w, \eta$

\begin{tabular}{|c|c|c|c|c|c|c|}
\hline \multicolumn{7}{|c|}{ The pessimistic value of $\mathrm{s}=0.2$} \\
\hline $\begin{array}{l}\text { Value of } \varepsilon_{1}, \varepsilon_{2}, \\
w_{p}=w_{\sigma}=w_{\delta}=w_{\tau} \\
=w_{\sigma}^{1}=w_{\delta}^{1}=w_{\tau}^{1}=w \\
\text { and } \\
\text { Desperation level } \\
\eta_{p}=\eta_{\sigma}=\eta_{\delta}=\eta_{\tau} \\
=\eta_{\sigma}^{1}=\eta_{\delta}^{1}=\eta_{\tau}^{1}=\eta\end{array}$ & $w=0.3$ & $\begin{array}{l}w=0.3 \\
\eta=0.6 \\
\varepsilon_{i}= \\
\left(U_{i}-L_{i}\right) \times 0.1\end{array}$ & $w=0.5$ & $\begin{array}{l}w=0.5 \\
\eta=0.5 \\
\varepsilon_{i}= \\
\left(U_{i}-L_{i}\right) \times 0.1\end{array}$ & $w=0.7$ & $\begin{array}{l}w=0.7 \\
\eta=0.2 \\
\varepsilon_{i}= \\
\left(U_{i}-L_{i}\right) \times 0.1\end{array}$ \\
\hline$x_{1}($ in $)$ & 0.3230761 & 0.3230720 & 0.3226488 & 0.3228958 & 0.3224620 & 0.3243104 \\
\hline$x_{2}($ in $)$ & 0.9823865 & 0.9839329 & 0.9800923 & 0.9829856 & 0.9790931 & 0.9906277 \\
\hline$x_{3}($ in $)$ & 1.806978 & 1.814461 & 1.802845 & 1.814461 & 1.801052 & 1.828308 \\
\hline$x_{4}($ in $)$ & 0.6244300 & 0.6246603 & 0.6245602 & 0.6246603 & 0.6246173 & 0.6242285 \\
\hline$C(X)(\$)$ & 0.1433088 & 0.1461895 & 0.1426818 & 0.0 .1461895 & 0.1424090 & 0.1495614 \\
\hline
\end{tabular}

Where $U_{i}$ and $L_{i}$ are upper and lower bound of respective objective and constraints

Table. 10 The optimum values of design variables for different values of $s, w, \eta$ 
Optimization of Welded Beam with Imprecise Load and Stress by Parameterized.. 605

\begin{tabular}{|l|l|l|l|l|l|l|}
\hline $\begin{array}{l}\text { Value of } \varepsilon_{1}, \varepsilon_{2}, \\
w_{p}=w_{\sigma}=w_{\delta}=w_{\tau} \\
=w_{\sigma}^{1}=w_{\delta}^{1}=w_{\tau}^{1}=w \\
\text { and } \\
\begin{array}{l}\text { Desperation level } \\
\eta_{p}=\eta_{\sigma}=\eta_{\delta}=\eta_{\tau} \\
=\eta_{\sigma}^{1}=\eta_{\delta}^{1}=\eta_{\tau}^{1}=\eta\end{array}\end{array}$ & $\begin{array}{l}w=0.3, \\
\eta=0.6 \\
\varepsilon_{i}= \\
\left(U_{i}-L_{i}\right) \times 0.1\end{array}$ & $w=0.5$, & $\begin{array}{l}w=0.5, \\
\eta=0.5 \\
\varepsilon_{i}= \\
\left(U_{i}-L_{i}\right) \times 0.1\end{array}$ & $w=0.7$, & $\begin{array}{l}w=0.7, \\
\eta=0.2 \\
\varepsilon_{i}= \\
\left(U_{i}-L_{i}\right) \times 0.1\end{array}$ \\
\hline$x_{1}(\mathrm{in})$ & 0.3216023 & 0.3233862 & 0.3216085 & 0.3232112 & 0.3216074 & 0.3246158 \\
\hline$x_{2}(\mathrm{in})$ & 0.9760721 & 0.9856260 & 0.9761017 & 0.9846832 & 0.9760963 & 0.9922898 \\
\hline$x_{3}(\mathrm{in})$ & 1.802017 & 1.819238 & 1.802058 & 1.817532 & 1.802050 & 1.831334 \\
\hline$x_{4}(\mathrm{in})$ & 0.6250552 & 0.6245107 & 0.6250529 & 0.6245641 & 0.6250534 & 0.6241353 \\
\hline$C(X)(\$)$ & 0.1426734 & 0.1468469 & 0.1426818 & 0.1464910 & 0.1426802 & 0.1493870 \\
\hline
\end{tabular}

Where $U_{i}$ and $L_{i}$ are upper and lower bound of respective objective and constraints.

Table.11 The optimum values of design variables for different values of $s, w, \eta$

\begin{tabular}{|c|c|c|c|c|c|c|}
\hline \multicolumn{7}{|c|}{ The pessimistic value of $\mathrm{s}=0.8$} \\
\hline $\begin{array}{l}\text { Value of } \varepsilon_{1}, \varepsilon_{2}, \\
w_{p}=w_{\sigma}=w_{\delta}=w_{\tau} \\
=w_{\sigma}^{1}=w_{\delta}^{1}=w_{\tau}^{1}=w \\
\text { and } \\
\text { Desperation level } \\
\eta_{p}=\eta_{\sigma}=\eta_{\delta}=\eta_{\tau} \\
=\eta_{\sigma}^{1}=\eta_{\delta}^{1}=\eta_{\tau}^{1}=\eta\end{array}$ & $w=0.3$ & $\begin{array}{l}w=0.3, \\
\eta=0.6 \\
\varepsilon_{i}= \\
\left(U_{i}-L_{i}\right) \times 0.1\end{array}$ & $w=0.5$ & $\begin{array}{l}w=0.5 \\
\eta=0.5 \\
\varepsilon_{i}= \\
\left(U_{i}-L_{i}\right) \times 0.1\end{array}$ & $w=0.7$ & $\begin{array}{l}w=0.7 \\
\eta=0.2 \\
\varepsilon_{i}= \\
\left(U_{i}-L_{i}\right) \times 0.1\end{array}$ \\
\hline$x_{1}($ in $)$ & 0.3211664 & 0.3237005 & 0.3216085 & 0.3235267 & 0.3217943 & 0.3249214 \\
\hline$x_{2}(i n)$ & 0.9737554 & 0.9873225 & 0.9761018 & 0.9863837 & 0.9770912 & 0.9939550 \\
\hline$x_{3}($ in $)$ & 1.797855 & 1.822309 & 1.802057 & 1.820608 & 1.803837 & 1.834365 \\
\hline$x_{4}($ in $)$ & 0.625188 & 0.6244147 & 0.6250529 & 0.6244678 & 0.6249963 & 0.6240418 \\
\hline$C(X)(\$)$ & 0.142041 & (6.1470916 & 0.1426818 & 0.1467933 & 0.1429521 & 0.1492130 \\
\hline
\end{tabular}


Where $U_{i}$ and $L_{i}$ are upper and lower bound of respective objective and constraints

From the above results it is clear whenever we chose fuzzy number and intuitionistic number for imprecise load and stress, deflection in welded beam design model, cost of welding is more optimized in fuzzy environment than the case of consideration of intuitionistic fuzzy environment.

\section{CONCLUSIONS}

In this paper, fuzzy and IFO approach have been used to solve IFNLPP with intuitionistic fuzzy resources as well as intuitionistic fuzzy numbers as coefficients which may be considered as triangular fuzzy and triangular intuitionistic fuzzy numbers. The proposed method is utilized with linear membership function to a welded beam design. At first step triangular fuzzy number and intuitionistic fuzzy number has been transformed into an interval number by interval approximation method. After that parametric interval valued functional form are created from that interval numbers so that the solution of the undertaken numerical problem can be solved by fuzzy and intuitionistic fuzzy optimization method given by Angelov [21]. As it has been seen that there is a few method in literature for solving IFNLP and the proposed method has been applied to a fully IFNLP problem so the method will be very beneficial and applicable for solving NLPP arising in other field of engineering in intuitionistic fuzzy environment .

\section{ACKNOWLEDGEMENT}

The research work of Mridula Sarkar is financed by Rajiv Gandhi National Fellowship (F1-17.1/2013-14-SC-wes-42549/(SA-III/Website)), Govt. of India.

\section{REFERENCES}

[1] K.M. Ragsdell, D.T. Phillips, Optimal design of a class of welded structures using geometric programming, ASME Journal of Engineering for Industries 98 (3)1976. 1021-1025, Series B.

[2] K. Deb, Optimal design of a welded beam via genetic algorithms, AIAA Journal 29 ,(11) 1991. 2013-2015.

[3] Deb, K., Pratap, A. and Moitra, S., Mechanical component design for multiple objectives using elitist non-dominated sorting GA. In Proceedings of the Parallel Problem Solving from Nature VI Conference,Paris, 16-20 September 2000, pp. 859-868. 
Optimization of Welded Beam with Imprecise Load and Stress by Parameterized.. 607

[4] Coello, C.A.C. 2000b. Use of a self-adaptive penalty approach for engineering optimization problems. Comput. Ind., 41: 113-127. DOI: 10.1016/S01663615(99)00046-9.

[5] Carlos A. Coello Coello, 'Solving Engineering Optimization Problems with the Simple Constrained Particle Swarm Optimizer', Informatica 32 (2008) 319-326.

[6] Reddy, M. J.; Kumar, D. N.,'An efficient single objective optimization algorithm based on swarm intelligence for engineering design' Engineering Optimization, Vol. 39, No. 1, January 2007, 49-68.

[7] Lee,K.S., Geem,Z.W. '”A new meta-heuristic algorithm for continuous engineering optimization: harmony search theory and practice' Comput. Methods Appl. Mech. Engrg. 194 (2005) 3902-3933.

[8] S. Kazemzadeh Azada, ,O. Hasançebia and O. K. Erol 'Evaluating efficiency of big-bang big-crunch algorithm in benchmark engineering optimization problems',,Int. J. Optim. Civil Eng., 2011; 3:495-505.

[9] Shuang Li,G. and Au,S.K. 'Solving constrained optimization problems via Subset Simulation''2010 4th International Workshop on Reliable Engineering Computing (REC 2010),doi:10.3850/978-981-08-5118-7 069.

[10] Mahdavi, M., Fesanghary , M., Damangir, E. , 'An improved harmony search algorithm for solving optimization problems" Applied Mathematics and Computation 188 (2007) 1567-1579.

[11] L. A. Zadeh, Fuzzy set, Information and Control, vol.8, no.3, pp.338-353, 1965.

[12] K. Atanassov, Intuitionistic fuzzy sets, Fuzzy sets and Systems, 20,87-96, 1986.

[13] M. Sarkar, T.K.Roy, Truss Design Optimization with Imprecise Load and Stress in Intuitionistic Fuzzy Environment, Journal of Ultra Scientist of Physical Sciences, 29(2) (2017)12-23,

[14] M. H. Shu, C. H. Cheng and J. R. Chang, Using intuitionistic fuzzy sets for fault-tree analysis on printed circuit board assembly, Microelectronics Reliability, 46(12) (2006)2139-2148,

[15] P. Grzegorzewski. Distances and orderings in a family of intuitionistic fuzzy numbers, EUSFLAT Conference, 223-227, 2003.

[16] H. B. Mitchell. Ranking-intuitionistic fuzzy numbers, International Journal of Uncertainty, Fuzziness and Knowledge-Based Systems, 12(3) (2004)377-386. 
[17] G. Nayagam, V. Lakshmana, G. Venkateshwari and G. Sivaraman. Ranking of intuitionistic fuzzy numbers, IEEE International Conference on Fuzzy Systems, IEEE World Congress on Computational Intelligence), (2008)19711974.

[18] H. M. Nehi. A new ranking method for intuitionistic fuzzy numbers, International Journal of Fuzzy Systems, 12(1) (2010)80-86.

[19] S. Rezvani. Ranking method of trapezoidal intuitionistic fuzzy numbers, Annals of Fuzzy Mathematics and Informatics, 5(3)(2013)515-523.

[20] H.J.Zimmermann, , Fuzzy linear programming with several objective function, Fuzzy sets and systems, 1(1978)46-55.

[21] Angelov, P.P. Optimization in intuitionistic fuzzy environment. Fuzzy Sets and Systems 86, 299-306, 1997.

[22] Dey.S.,Roy ,T.K., Parameterized Intuitionistic Fuzzy Optimization Method and its Applicatin to Structural Design. International Journal of Computer Science \&Engineering Technology, 8(03), 2017, 72-84.

[23] Singh.B.,Sarkar M. ,Roy ,T.K., Intuitionistic Fuzzy Optimization Of Truss Design: A Comparative Study. International Journal of Computer and Organization Trends, 37(1), 2016, 25-33. 